ARTICLE

DOI: $10.1038 / s 41467-017-02629-3$

\title{
Electric-field control of anomalous and topological Hall effects in oxide bilayer thin films
}

\author{
Yuki Ohuchi', Jobu Matsuno (D) 2, Naoki Ogawa², Yusuke Kozuka', Masaki Uchida (D) ${ }^{1}$,
} Yoshinori Tokura ${ }^{1,2} \&$ Masashi Kawasaki ${ }^{1,2}$

One of the key goals in spintronics is to tame the spin-orbit coupling (SOC) that links spin and motion of electrons, giving rise to intriguing magneto-transport properties in itinerant magnets. Prominent examples of such SOC-based phenomena are the anomalous and topological Hall effects. However, controlling them with electric fields has remained unachieved since an electric field tends to be screened in itinerant magnets. Here we demonstrate that both anomalous and topological Hall effects can be modulated by electric fields in oxide heterostructures consisting of ferromagnetic $\mathrm{SrRuO}_{3}$ and nonmagnetic $\mathrm{SrlrO}_{3}$. We observe a clear electric field effect only when $\mathrm{SrlrO}_{3}$ is inserted between $\mathrm{SrRuO}_{3}$ and a gate dielectric. Our results establish that strong $\mathrm{SOC}$ of nonmagnetic materials such as $\mathrm{SrlrO}_{3}$ is essential in electrical tuning of these Hall effects and possibly other SOC-related phenomena.

\footnotetext{
${ }^{1}$ Department of Applied Physics and Quantum-Phase Electronics Center (QPEC), University of Tokyo, Tokyo 113-8656, Japan. ${ }^{2}$ RIKEN Center for Emergent Matter Science (CEMS), Wako, Saitama 351-0198, Japan. Correspondence and requests for materials should be addressed to J.M. (email: matsuno@riken.jp)
} 
$\mathrm{P}$ rofound implications for the electric field control of spin states have been revealed through magneto-transport properties arising from the spin-orbit coupling (SOC) ${ }^{1}$. Such properties have been attracting great interest as foundations for high-density and low-power-consumption spintronic devices because the properties coupled to the spin states enable spin manipulation without magnetic field variation or large current injection. Among the magneto-transport processes, of interest here is the intrinsic anomalous Hall effect ${ }^{2}$ (AHE), which is related to magnetization $(M)$ in Hall resistivity $\left(\rho_{\mathrm{AHE}}=R_{\mathrm{S}} M\right)$. In some itinerant ferromagnets such as $\mathrm{SrRuO}_{3}{ }^{3}, 4$, the proportionality factor $\left(R_{\mathrm{S}}\right)$ is governed by $k$-space monopoles, i.e., singularities originating from band-crossings gapped by SOC; not $M$ but $R_{\mathrm{S}}$ is potentially tuneable by electric field there. Another intriguing example is the topological Hall effect (THE) originating from scalar spin chirality concomitant with non-coplanar spin structures such as in a frustrated pyrochlore magnet ${ }^{5}$ and in metallic magnets characterized by a non-zero skyrmion number $\left(N_{\text {sk }}\right)^{6-9}$. In the case of $N_{\text {sk }}=1$, the topologically protected spin swirling texture is called magnetic skyrmion ${ }^{6}, 10,11$. Despite mounting interest in electrical control of these Hall effects, however, their control in itinerant magnets has been elusive in conventional field-effect structures, that is, magnetic materials adjacent to gate dielectrics. Recent discovery of the THE in an oxide heterostructure composed of $\mathrm{SrRuO}_{3}$ and $\mathrm{SrIrO}_{3}{ }^{12}$ brings an opportunity to develop electrical control because the strong SOC in $\mathrm{SrIrO}_{3}{ }^{13}$ induces a SOC-related phenomenon in neighboring ferromagnetic $\mathrm{SrRuO}_{3}$. In addition to the intrinsic AHE inherent to $\mathrm{SrRuO}_{3}{ }^{3}$, the combination of the strong SOC and the broken inversion symmetry gives rise to substantial Dzyaloshinskii-Moriya interaction (DMI) ${ }^{6}, 14$ and the concomitant THE; the underlying spin texture has been deduced to be the interface-driven skyrmion ${ }^{12}$. In view of the fact that ultrathin films of itinerant magnets have been a playground for electrical manipulation of spin states ${ }^{1,15-19}$, an electric field applied to the ultrathin heterostructure may provoke effective manipulation of the spin state contending with screening effects by itinerant electrons of $\mathrm{SrRuO}_{3}$. Furthermore, we can expect that the strong SOC in $\mathrm{SrIrO}_{3}$ plays a positive role at the interface as well because both AHE and THE are magneto-transport phenomena driven by SOC.

Here, we show the clear modulation of both AHE and THE by an electric field in the heterostructure of $\mathrm{SrRuO}_{3} / \mathrm{SrIrO}_{3} / \mathrm{SrTiO}_{3}$ from magneto-transport and magneto-optic Kerr effect (MOKE) measurements. The electrical modulation is effective only when $\mathrm{SrIrO}_{3}$ is inserted between $\mathrm{SrRuO}_{3}$ and a gate dielectric, i.e., $\mathrm{SrTiO}_{3}$. This indicates the essential role of the strong SOC in nonmagnetic materials for the electrical tuning of these Hall effects and possibly other SOC-related phenomena.

\section{Results}

Stacking-order dependent electric field control. Five-unit-cell $\mathrm{SrRuO}_{3}$ thin films were epitaxially grown on $\mathrm{SrTiO}_{3}(001)$ substrates by pulsed laser deposition in three stacking orders with $\mathrm{SrIrO}_{3}$ (see Methods): a single layer of $\mathrm{SrRuO}_{3}$ (SRO5/Sub), a bilayer of $\mathrm{SrRuO}_{3}$ and 2-unit-cell $\mathrm{SrIrO}_{3}$ (SIO2/SRO5/Sub), and that with the inverted deposition order (SRO5/SIO2/Sub, Fig. 1a). We first take a look at basic transport and magnetic properties before we go into the Hall effects under an applied electric field (Fig. 1b). In Fig. 1c, d, we show the temperature dependence of longitudinal resistivity $\left(\rho_{x x}\right)$ and magnetization perpendicular to the film plane in the three samples. All of them show metallic conduction and ferromagnetic magnetization whose Curie temperatures $\left(T_{\mathrm{C}}\right)$ are lower than the bulk value $(160 \mathrm{~K})^{3}$. These transport and magnetic properties are consistent with previously reported ultrathin $\mathrm{SrRuO}_{3}$ films ${ }^{20}$. Figure le shows anomalous Hall conductivity $\left(\sigma_{\mathrm{AHE}}\right)$ as a function of magnetization with temperature as a control parameter. The $\sigma_{\mathrm{AHE}}$ of the three samples behave the same way; when the magnetization gets larger, the sign of $\sigma_{\mathrm{AHE}}$ is inverted from positive to negative. This sign inversion is consistent with the previous experiments ${ }^{4,12}$, manifesting the above-mentioned band anti-crossing singularities of $\mathrm{SrRuO}_{3}$. Conversely, such temperature dependence in AHE implies the potential for electrical control of AHE through the modification of the SOC-induced band.

In contrast to the similarities of the basic transport and magnetic properties among the three samples, a clear difference emerges in electric field effect (see Methods). The sums of anomalous and topological Hall resistivities $\left(\rho_{\mathrm{AHE}}+\rho_{\mathrm{THE}}\right)$ under applied electric fields are shown in Figs. $1 \mathrm{f}-\mathrm{h}$ as a function of external magnetic field $(B)$. The $B$ dependence of $\rho_{\mathrm{AHE}}$ corresponds to that of magnetization normal to the film plane, whereas $\rho_{\text {THE }}$ shows up with the formation of the topological spin texture as exemplified by the peak at around $3 \mathrm{~T}$ in Fig. 1h. The sum of these Hall components is obtained by subtracting the $B$ linear ordinary Hall term $\left(R_{\mathrm{H}} B\right)$ from the Hall resistivity $\left(\rho_{y x}\right)$. Both in SRO5/Sub (Fig. 1f) and in SIO2/SRO5/Sub (Fig. 1g), $\rho_{\mathrm{AHE}}+\rho_{\mathrm{THE}}$ is almost unchanged by the applied electric field. However, $\rho_{\mathrm{AHE}}+\rho_{\mathrm{THE}}$ is obviously changed in SRO5/SIO2/Sub (Fig. 1h). This striking stacking-order dependence suggests that the electric field to the $\mathrm{SrRuO}_{3}-\mathrm{SrIrO}_{3}$ interface causes the noticeable modulation. The thinness of the $\mathrm{SrIrO}_{3}$ layer compared with the $\mathrm{SrRuO}_{3}$ layer contributes to the preferable modulation in SRO5/SIO2/Sub because the interface in SRO5/ $\mathrm{SIO} 2 / \mathrm{Sub}$ is closer to the gate dielectric than that in SIO2/SRO5/ Sub (see Supplementary Figure 1 and Supplementary Note 1). Hereafter, we focus on the control in the SRO5/SIO2/Sub sample.

Electric-field modulation of AHE. In the structure of SRO5/ $\mathrm{SIO} 2 / \mathrm{Sub}$, the large electric field modulation gives rise to the sign inversion of $\mathrm{AHE}$ without changing temperature. Magnetic-field dependences of $\rho_{\mathrm{AHE}}+\rho_{\mathrm{THE}}$ under different electric fields at $30 \mathrm{~K}$ are shown in Fig. 2a-c. Except for the topological Hall term that shows a peak at around $0.8 \mathrm{~T}$, the data are dominated by the anomalous Hall contribution. In particular, we can consider the value at high magnetic field such as $2 \mathrm{~T}$ to be totally derived from $\rho_{\mathrm{AHE}}$ since all the spins are ferromagnetically aligned without forming any topological spin textures. The sign of $\rho_{\mathrm{AHE}}$ above the saturation field is inverted from negative to positive when the gate voltage is varied from negative $(-180 \mathrm{~V})$ to positive $(200 \mathrm{~V})$, while $\rho_{\mathrm{AHE}}$ is close to vanish under zero bias. This sign inversion indicates the sign reversal of the proportionality factor $R_{\mathrm{S}}$ because MOKE measurements reveal that only a minor fraction of $M$, $<10 \%$ of the magnetization, is electrically modulated (see Supplementary Figure 2 and Supplementary Note 2). Such electrical sign inversion has never been observed in plain films of itinerant magnets including $\mathrm{SrRuO}_{3}$.

As shown in Fig. 2d, the tendency of AHE change is nearly temperature independent; the positive (negative) bias voltage increases (decreases) $\rho_{\mathrm{AHE}}$ regardless of the sign of AHE. Since singularities in the band structure of $\mathrm{SrRuO}_{3}$ originate from band anti-crossings gapped by SOC, we speculate that the electric field control of AHE is ascribed to the redistribution of the singularities caused by the variation of SOC. The pronounced controllability in SRO5/SIO2/Sub compared with its absence in SIO2/SRO5/Sub suggests that the electric field from $\mathrm{SrIrO}_{3}$ side induces certain modification of SOC in the bilayer even if it has itinerant carrier density as high as $10^{22} \mathrm{~cm}^{-3}$ in the magnetic $\mathrm{SrRuO}_{3}$ layer. This is in sharp contrast to the case of chemically doped $\mathrm{EuTiO}_{3}{ }^{21}$, where carrier-density variation inverts the sign 
a

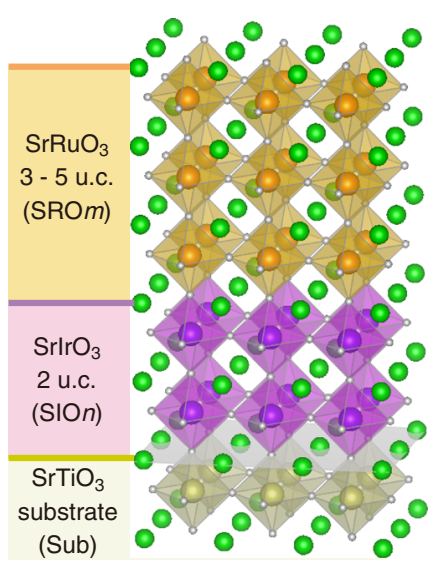

b

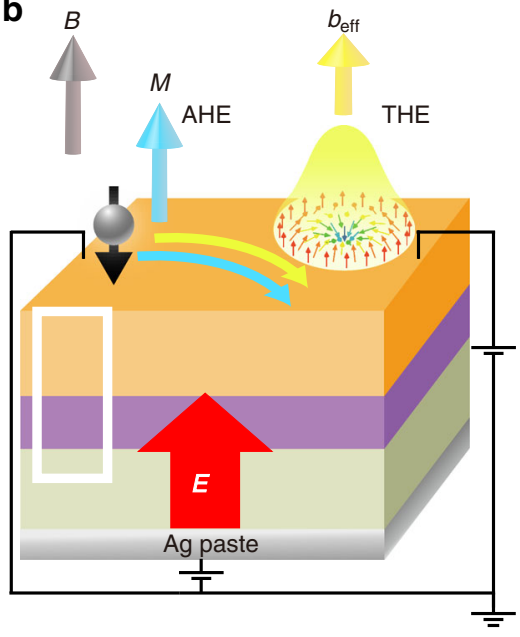

C

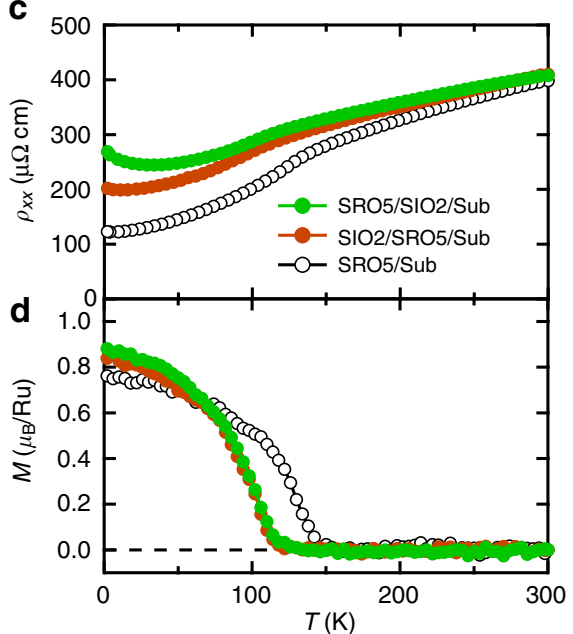

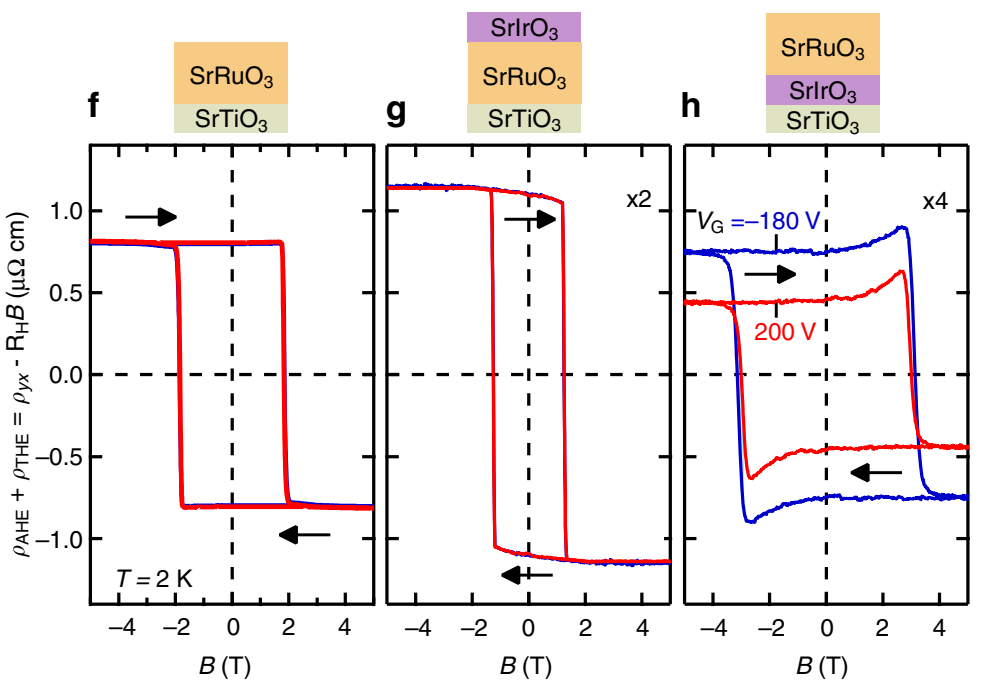

e

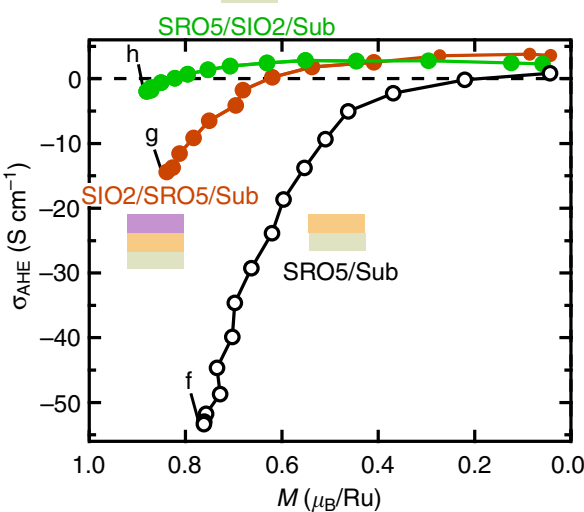

Fig. 1 Structure and basic physical properties of samples. a Schematics of the $\mathrm{SrRuO}_{3}-\mathrm{SrlrO}_{3}$ bilayer film, where $\mathrm{SrlrO}_{3}$ is inserted between $\mathrm{SrRuO}_{3}$ and $\mathrm{SrTiO}_{3}$ substrate (SROm/SIO2/Sub, $m=3-5$ unit cells). Green, orange, purple, beige, and gray atoms represent $\mathrm{Sr}, \mathrm{Ru}$, Ir, Ti, and O, respectively, in the crystal structure, which is drawn using VESTA ${ }^{38}$. b Schematics of the magneto-transport properties observed in SROm/SIO2/Sub under the application of a gate electric field ( $E$ ): Anomalous Hall effect (AHE) generated by magnetization $(M)$ and topological Hall effect (THE) driven by fictitious magnetic field $\left(b_{\text {eff }}\right)$ in the case of skyrmion formation. White box corresponds to the schematics in $\mathbf{a}$. Temperature $(T)$ dependence of longitudinal resistivity $\left(\rho_{x x}, \mathbf{c}\right)$ and out-of-plane magnetization measured at $0.1 \mathrm{~T}(\mathrm{M}, \mathbf{d})$ for SRO5/Sub, SIO2/SRO5/Sub, and SRO5/SIO2/Sub. e Anomalous Hall conductivity $\left(\sigma_{\mathrm{AHE}}\right)$ as a function of magnetization $(M)$. $\mathbf{f}-\mathbf{h}$ The sum of anomalous and topological Hall resistivity $\left(\rho_{\mathrm{AHE}}+\rho_{\mathrm{THE}}\right)$ at $2 \mathrm{~K}$ as a function of external magnetic field (B) under application of gate bias $V_{G}=-180 \mathrm{~V}$ (blue lines) and $200 \mathrm{~V}$ (red lines). At the lowest temperature $(T=2 \mathrm{~K}$ ), the largest modulation of carrier density can be realized due to the quantum paraelectric nature of $\mathrm{SrTiO}_{3}$ substrates. On top, respective sample structure is shown. $\rho_{\mathrm{AHE}}+\rho_{\mathrm{THE}}$ is deduced by subtracting a $B$-linear ordinary Hall component $\left(R_{\mathrm{H}} B\right)$ from the Hall resistivity $\left(\rho_{y x}\right)$. Black arrows indicate the sweep direction of $B$

of AHE accompanied by the shift of Fermi energy $\left(E_{\mathrm{F}}\right)$. The insertion of the $\mathrm{SrIrO}_{3}$ layer makes a more important contribution to the AHE modulation than the $E_{\mathrm{F}}$ shift in $\mathrm{SrRuO}_{3}$ does. The controllability may partly rely on the lower carrier density of inserted semimetallic $\mathrm{SrIrO}_{3}$, the order of $10^{19} \mathrm{~cm}^{-322}$.

In order to clarify the effectiveness of the heterointerface close to the gate dielectric, we also fabricate the heterostructure with thicker (5 unit cells) $\mathrm{SrIrO}_{3}$ (SRO5/SIO5/Sub, see Supplementary Figures 3-5 and Supplementary Notes 3 and 4). Figure 2e shows magnetic field dependences of $\rho_{\mathrm{AHE}}+\rho_{\mathrm{THE}}$ under different electric fields at $30 \mathrm{~K}$ in SRO5/SIO5/Sub, where the change of $\rho_{\mathrm{AHE}}$ is apparently suppressed. In Fig. 2f, we compare the variation of $\rho_{\mathrm{AHE}}\left(\Delta \rho_{\mathrm{AHE}}=\rho_{\mathrm{AHE}}\left(V_{\mathrm{G}}=200 \mathrm{~V}\right)-\rho_{\mathrm{AHE}}\left(V_{\mathrm{G}}=-180\right.\right.$ $\mathrm{V})$ ) in SRO5/SIO5/Sub with that in SRO5/SIO2/Sub, where we can clearly see the suppression of the modulation in the thicker $\mathrm{SrIrO}_{3}$ sample within all the measured temperatures. We attribute this suppression to the significant screening of the electric field within the thicker $\mathrm{SrIrO}_{3}$ layer.

Electric-field modulation of THE. THE is also modulated by electric field in the structure of SRO5/SIO2/Sub. In order to evaluate $\rho_{\mathrm{THE}}$ from magneto-transport measurement, $\rho_{\mathrm{AHE}}$ has to be subtracted from $\rho_{\mathrm{AHE}}+\rho_{\mathrm{THE}}$. Since MOKE is proportional to magnetization as anticipated from its perturbative nature and also as experimentally verified in the previous report ${ }^{12}$, MOKE under applied electric field can be utilized as the reference of $\rho_{\mathrm{AHE}}$, which is also proportional to magnetization at a constant temperature. The magnetic field dependences of Kerr rotation are shown by the broken lines in Fig. 2a-c. Each Kerr rotation is normalized by $\rho_{\mathrm{AHE}}$ at high magnetic field under each applied electric field; the normalized curves represent the $B$ dependence of $\rho_{\mathrm{AHE}}$. By subtracting them from $\rho_{\mathrm{AHE}}+\rho_{\mathrm{THE}}$, we deduced $\rho_{\mathrm{THE}}$ 


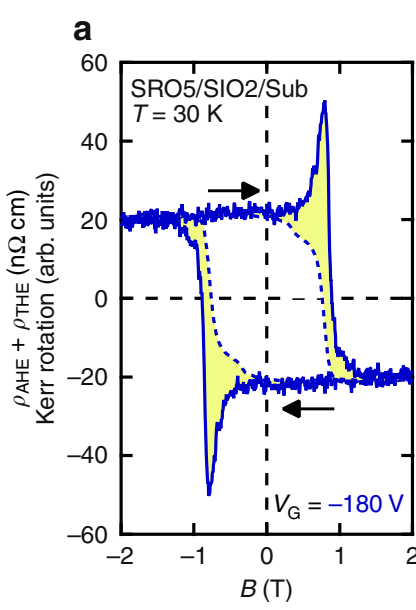

b

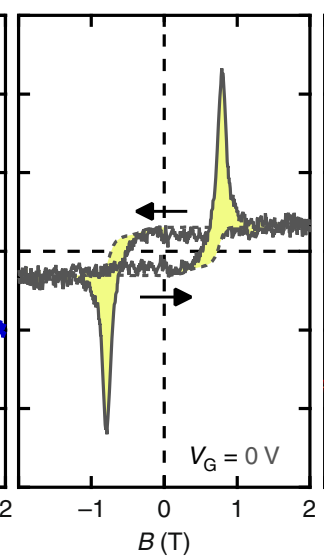

c

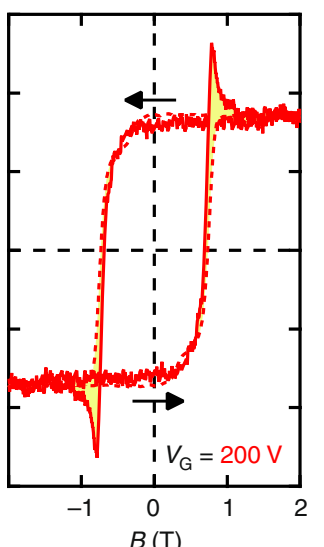

d

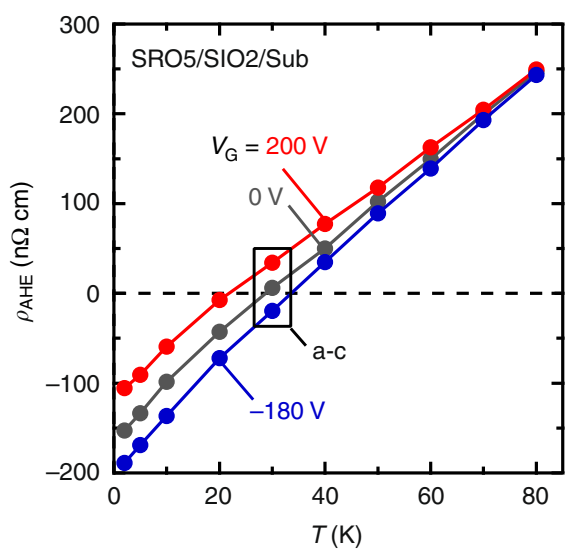

e

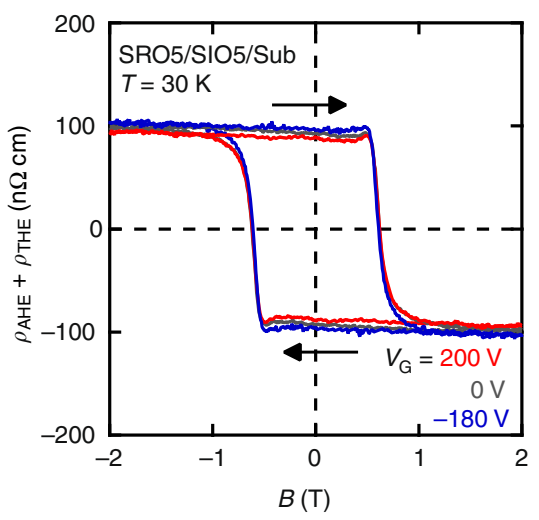

f

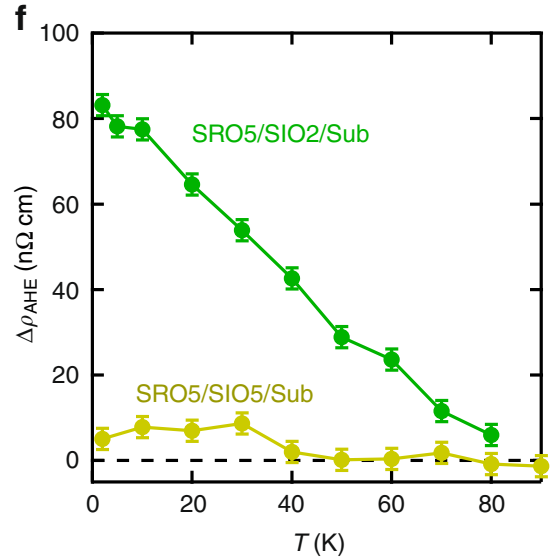

Fig. 2 Electric-field control of anomalous Hall effect. Magnetic-field $(B)$ dependence of anomalous and topological Hall resistivities $\left(\rho_{\mathrm{AHE}}+\rho_{\mathrm{THE}}\right.$ solid lines) at $30 \mathrm{~K}$ under gate voltage $V_{\mathrm{G}}=-180 \mathrm{~V}(\mathbf{a}), 0 \mathrm{~V}(\mathbf{b})$, and $200 \mathrm{~V}(\mathbf{c})$ for SRO5/SIO2/Sub. Magneto-optic Kerr rotation as a function of $B$ under the same gate bias is also shown by broken lines in each panel. Yellow colored regions correspond to $\rho_{\text {THE. }}$ Black arrows indicate the sweep direction of $B$. $\mathbf{d}$ Temperature $\left(T\right.$ ) dependence of $\rho_{\text {AHE }}$ under $V_{\mathrm{G}}=-180 \mathrm{~V}$ (blue), $0 \mathrm{~V}$ (gray) and $200 \mathrm{~V}$ (red). The black box corresponds to the data in a-c. e $B$ dependence of $\rho_{\text {AHE }}+\rho_{\text {THE }}$ at $30 \mathrm{~K}$ under $V_{\mathrm{G}}=-180 \mathrm{~V}$ (blue), $0 \mathrm{~V}$ (gray), and $200 \mathrm{~V}$ (red) for SRO5/SIO5/Sub. $\mathbf{f} T$ dependence of variation in anomalous Hall resistivity $\left(\Delta \rho_{\mathrm{AHE}}\right)$ between $V_{\mathrm{G}}=200 \mathrm{~V}$ and $V_{\mathrm{G}}=-180 \mathrm{~V}$ for SRO5/SIO5/Sub (yellow) and SRO5/SIO2/Sub (green). Error bars include the uncertainty of the sample thickness and of electrical measurement

(yellow colored regions in Fig. 2a-c). Figure 3a shows the electric field-controlled peak of $\rho_{\mathrm{THE}}$ around $B=0.8 \mathrm{~T}$. The electric field also tunes the range of magnetic field where finite $\rho_{\text {THE }}$ appears; when the positive (negative) gate voltage is applied, $\rho_{\mathrm{THE}}$ gets smaller (larger) and the magnetic field region gets shrunk (enlarged). The variation in peak of $\rho_{\mathrm{THE}}\left(\Delta \rho_{\mathrm{THE}}=\rho_{\mathrm{THE}}\left(V_{\mathrm{G}}=\right.\right.$ $\left.200 \mathrm{~V})-\rho_{\mathrm{THE}}\left(V_{\mathrm{G}}=-180 \mathrm{~V}\right)\right)$ normalized by zero-bias value $\left(\Delta \rho_{\mathrm{THE}} / \rho_{\mathrm{THE}}(0 \mathrm{~V})\right)$ is as large as $55 \%$. The sign of THE remains the same during the sign inversion of AHE by electric field, supporting that the origins of AHE and THE are clearly distinct from each other as previously indicated ${ }^{12}$.

Assuming the skyrmion formation in this heterostructure, we discuss the relation of this THE modulation with skyrmion length scale. $\rho_{\text {THE }}$ is an outgrowth of ordinary Hall effect under fictitious magnetic field $\left(b_{\text {eff }}\right)$ caused by the non-coplanar spin texture of skyrmion ${ }^{6}$. Since one skyrmion generates one flux quantum $\left(\Phi_{0}=h / e\right), \rho_{\mathrm{THE}}$ is described as a function of skyrmion density $\left(n_{\mathrm{sk}}\right)$ :

$$
\rho_{\mathrm{THE}}=P R_{\mathrm{H}} b_{\mathrm{eff}}=P R_{\mathrm{H}} n_{\mathrm{sk}} \Phi_{0}
$$

where $P$ is spin polarization of conduction electrons in $\mathrm{SrRuO}_{3}$. Ordinary Hall coefficient $R_{\mathrm{H}}$ is inversely proportional to carrier density. The change of $P$ or $R_{\mathrm{H}}$ is negligible because $E_{\mathrm{F}}$ in $\mathrm{SrRuO}_{3}$ remains almost unchanged judging from the variation of $\rho_{x x}$ as small as the order of $1 \%$ as discussed later. Therefore, the modulation of $\rho_{\mathrm{THE}}$ indicates the change of $n_{\mathrm{sk}}$. The skyrmion size, estimated by $r_{\mathrm{sk}} \cong 1 / \sqrt{n_{\mathrm{sk}}}$, is varied from $15 \mathrm{~nm}\left(V_{\mathrm{G}}=200 \mathrm{~V}\right)$ to $12 \mathrm{~nm}\left(V_{\mathrm{G}}=-180 \mathrm{~V}\right)$ adopting $P=-9.5 \%$ as a typical value of $\mathrm{SrRuO}_{3}{ }^{23}$ and $R_{\mathrm{H}}$ evaluated from the $B$-linear component of Hall resistivity under $V_{\mathrm{G}}=0 \mathrm{~V}$ at $30 \mathrm{~K}$. Since $r_{\mathrm{sk}}$ is proportional to the ratio of ferromagnetic interaction $J$ to DMI $D$, or $J / D^{6}$, the positive (negative) gate voltage should increase (decrease) $J / D$. The controlling knob of $\rho_{\text {THE }}$ is thought to be $D$ rather than J; the variation of $J$ is negligible because the applied electric field hardly varies $T_{\mathrm{C}}$, which can be evaluated by a kink structure in $\rho_{x x}{ }^{3}$ (see Supplementary Figure 6 and Supplementary Note 5). The realspace observation of such electrical control of skyrmion size would be one of the future challenges.

We examine the other possibilities of the underlying spin texture. One of the candidates is the conical spin structure, which is topologically trivial and is reported to give rise to THE-like signal ${ }^{24}$. In our case, this can be excluded since the spin structure is dominated by conventional ferromagnetic phase with finite anisotropy as exemplified by open hysteresis loops in magnetization (see Supplementary Figure 2). The possibility of noncoplanar spin structures stabilized only under geometrical frustration $^{5,25}$ can also be excluded because such frustration is apparently absent in our heterostructure. We then focus on a 
a

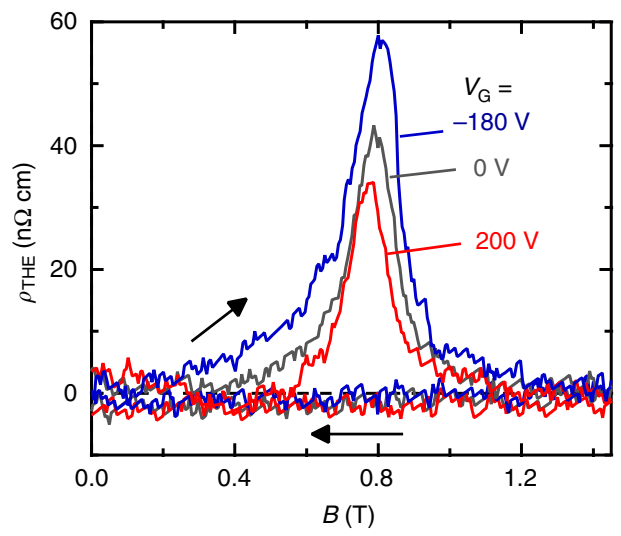

b

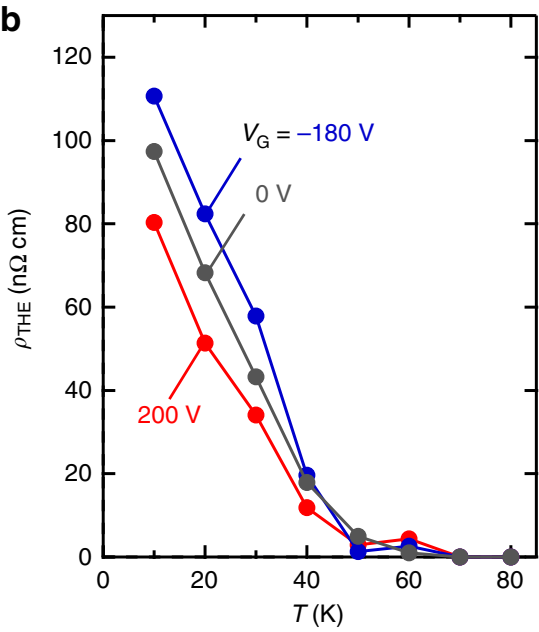

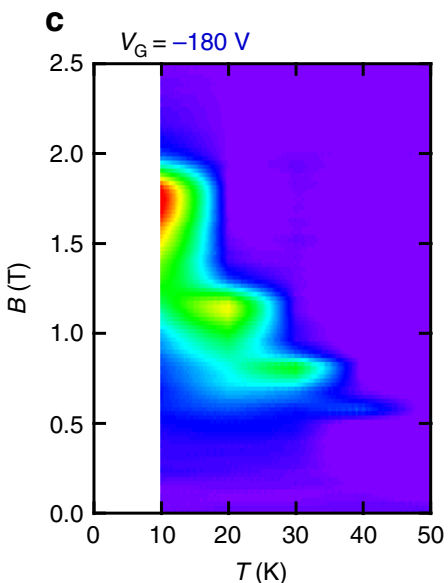
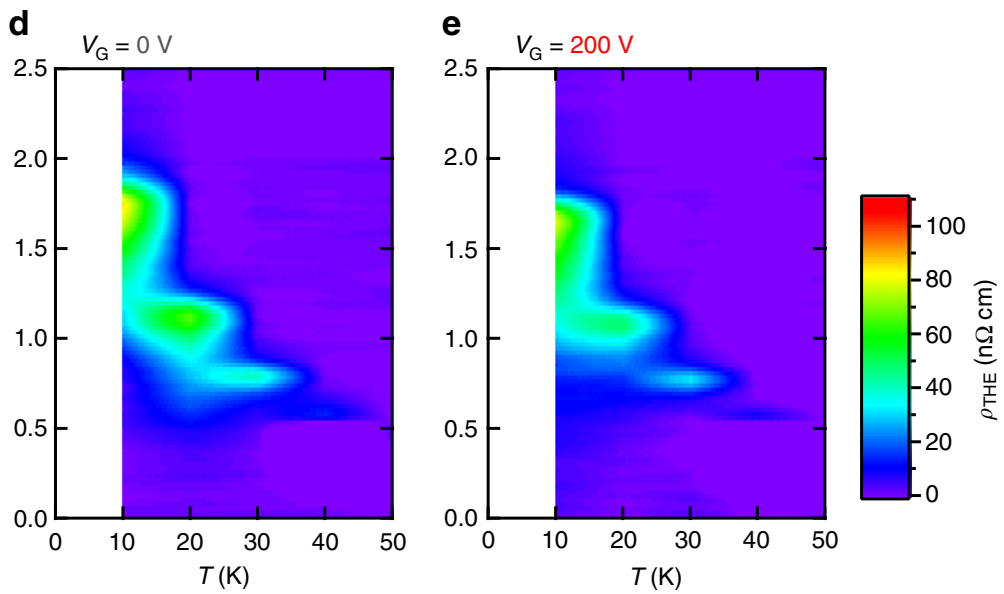

Fig. 3 Electric-field control of topological Hall effect. a Topological Hall resistivity $\left(\rho_{\text {THE }}\right)$ at $30 \mathrm{~K}$ as a function of external magnetic field $(B)$ under $V_{\mathrm{G}}=$ $-180 \mathrm{~V}$ (blue line), $0 \mathrm{~V}$ (gray line) and $200 \mathrm{~V}$ (red line) for SRO5/SIO2/Sub. Black arrows indicate the sweep direction of $B$. b Temperature ( $T$ ) dependence of $\rho_{\text {THE }}$ under gate bias. Color map of $\rho_{\text {THE }}$ in the $T-B$ plane under $V_{\mathrm{G}}=-180 \mathrm{~V}$ (c), $0 \mathrm{~V}$ (d) and $200 \mathrm{~V}(\mathbf{e})$

topological spin texture other than skyrmion e.g., biskyrmion or meron, of which topological number $\left(N_{\text {sk }}\right)$ is not unity $9,26,27$. The emergent fictitious magnetic field can thus be generalized as $n_{\text {topo }} N_{\text {sk }} \Phi_{0}$, where $n_{\text {topo }}$ is the density of the topological spin texture. Since $N_{\text {sk }}$ is constant inherent in the topological spin structures and hence independent of the gate bias, the electric field modulation of $\rho_{\text {THE }}$ is attributed to the modulation of $n_{\text {topo }}$. In order to realize topologically nontrivial spin structures in $\mathrm{SrRuO}_{3}$, the interface-driven DMI is required as discussed in the above-mentioned skyrmion case. The ratio of $J / D$ is inversely correlated with $n_{\text {topo }}$ for any type of topological spin textures. The consequence therefore remains the same; positive (negative) gate voltage should increase (decrease) $J / D$.

Considering that the variation of DMI is attributed to the modification of SOC at the interface, both modulations of AHE and THE are brought about by the same origin; this appears in the temperature dependence. The tendency of $\rho_{\text {THE }}$ change is independent of temperature as shown in Fig. $3 \mathrm{~b}$. The expansion of the observable magnetic field region under negative electric field is discerned at every temperature below $40 \mathrm{~K}$ (Fig. 3c-e). These indicate that the obtained modulation of THE is independent of the sign of AHE, which is inverted at $30 \mathrm{~K}$. The temperature independent trend of the electric field effect on THE is in accord with that on AHE (Fig. 2d), implying the common origin of SOC variation. In fact, the MOKE measurements indicate that the coercive force $\left(H_{\mathrm{C}}\right)$ is shifted from $0.73 \mathrm{~T}\left(V_{\mathrm{G}}=\right.$ $200 \mathrm{~V}$ ) to $0.76 \mathrm{~T}\left(V_{\mathrm{G}}=-180 \mathrm{~V}\right)$ at $30 \mathrm{~K}$ (see Supplementary
Figure 2 and Supplementary Note 2). This shift of $H_{\mathrm{C}}$ also supports the modification of SOC because the magnetic anisotropy originates from SOC.

\section{Discussion}

The observed modulations of the AHE and THE are not simply explained by the variation of carrier density. In order to show this clearly, the variations of $\operatorname{AHE}\left(\Delta \rho_{\mathrm{AHE}}\right)$ and $\operatorname{THE}\left(\Delta \rho_{\mathrm{THE}} / \rho_{\mathrm{THE}}(0\right.$ $\mathrm{V})$ ) with different thickness of $\mathrm{SrRuO}_{3}$ films are shown in Fig. $4 \mathrm{a}$, b, respectively, as a function of $\rho_{x x}$ variation normalized by zerobias value $\left(\Delta \rho_{x x} / \rho_{x x}(0 \mathrm{~V})\right)$ at various temperatures (see Supplementary Figures 7-9 and Supplementary Notes 6-8 for detailed transport and magnetic properties). $\Delta \rho_{x x} / \rho_{x x}(0 \mathrm{~V})$ ranges from $1.2 \%$ in SRO5/SIO2/Sub to $6.6 \%$ in SRO3/SIO2/Sub at $10 \mathrm{~K}$. Since $\rho_{x x}$ is inversely proportional to carrier density $(n)$, the order of magnitude is consistent with the expected electron accumulation by a $\mathrm{SrTiO}_{3}$-back-gate transistor (Supplementary Note 8 ). $\Delta \rho_{\mathrm{AHE}}$ in bilayers indicates that the gate-bias control of AHE has the specific tendency regardless of the film thickness. The same plots of $\mathrm{SrRuO}_{3}$ single layers ( $\left.\mathrm{SRO} / \mathrm{Sub}\right)$ are also depicted for comparison. They have negligible change in AHE, while their $\rho_{x x}$ variation is in the same range with that in bilayers with the same $\mathrm{SrRuO}_{3}$ thickness. As already pointed out, these results demonstrate the importance of inserting the $\mathrm{SrIrO}_{3}$ layer rather than the $E_{\mathrm{F}}$ shift in $\mathrm{SrRuO}_{3}$. According to the equation (1), $\rho_{\mathrm{THE}}$ is proportional not only to $n_{\mathrm{sk}}$ (or $n_{\text {topo }} N_{\mathrm{sk}}$ ) but also to $R_{\mathrm{H}}$. We show the calculated variation of $\rho_{\mathrm{THE}}$ contributed from $R_{\mathrm{H}}$, i.e., $n$ 

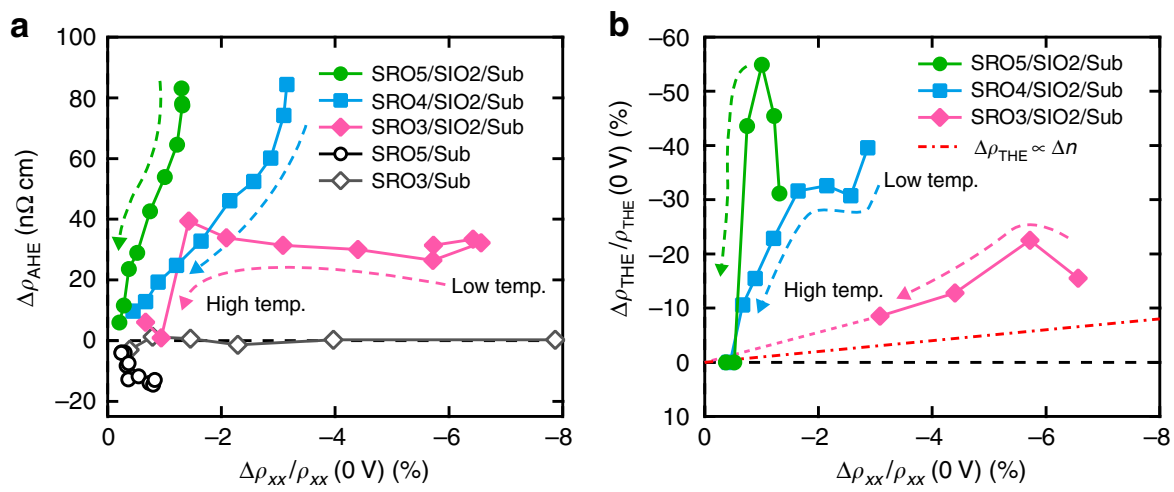

Fig. 4 Qualitative analyses of modulations in AHE and THE. a Difference in anomalous Hall resistivity $\left(\Delta \rho_{\mathrm{AHE}}\right)$ between $V_{\mathrm{G}}=200 \mathrm{~V}$ and $V_{\mathrm{G}}=-180 \mathrm{~V}$ as a function of longitudinal resistivity variation ratio $\left(\Delta \rho_{x x} / \rho_{x x}(\mathrm{OV})\right)$ at temperatures ranging from $2 \mathrm{~K}$ to $80 \mathrm{~K}$. $\mathbf{b}$ Modulation ratio of topological Hall resistivity $\left(\Delta \rho_{\mathrm{THE}} / \rho_{\mathrm{THE}}(\mathrm{OV})\right)$ as a function of $\Delta \rho_{\mathrm{x} x} / \rho_{x \mathrm{x}}(\mathrm{OV})$. Red dash-dotted line is the calculated variation where gate bias only changes carrier density $(\Delta n)$. Pink dotted line is the guide to the eyes. Broken arrows indicate temperature variation

variation $\left(\Delta R_{\mathrm{H}}\right.$ i.e. $\left.\Delta n=n\left(V_{\mathrm{G}}=200 \mathrm{~V}\right)-n\left(V_{\mathrm{G}}=-180 \mathrm{~V}\right)\right)$ with the red dash-dotted line in Fig. $4 \mathrm{~b}$, assuming small $\Delta n\left(\Delta R_{\mathrm{H}} \propto\right.$ $\Delta n / n^{2}$, i.e., $\left.\Delta \rho_{\mathrm{THE}} \propto \Delta n\right)$ and $V_{\mathrm{G}}$-independent electron mobility. All the modulations in heterostructures are located far above the red dash-dotted line. Therefore, most of the observed $\Delta \rho_{\mathrm{THE}}$ is attributed to the change in size of the topological spin texture, i.e., DMI. These pronounced modulations of the AHE and THE are both achieved not by carrier-density variation in $\mathrm{SrRuO}_{3}$ but by modification of SOC at the interface of $\mathrm{SrRuO}_{3}$ and $\mathrm{SrIrO}_{3}$.

Recent theoretical calculations ${ }^{28,29}$ have revealed that the DMI is enormously affected by the singularities in band structures as well as the AHE, although the precise dependencies differ between them. This has been confirmed in itinerant chiral magnets $^{30}$ and a semiconducting Dirac electron system ${ }^{31}$. We speculate that our bilayers have some band deformation when applying an electric field to the interface. In both plots in Fig. 4, thinner $\mathrm{SrRuO}_{3}$ films exhibit smaller variation in spite of the larger $\Delta \rho_{x x} / \rho_{x x}(0 \mathrm{~V})$. This thickness dependence is probably attributed to band structure deformation as observed in the thin limit of $\mathrm{SrRuO}_{3}{ }^{32}$; the density of states near $E_{\mathrm{F}}$ shrinks as $\mathrm{SrRuO}_{3}$ gets thinner.

One of the plausible pathways in the electric field-induced change of SOC is through the interface potential gradient, which is observed as the electrical control of Rashba-type band splitting in a semiconductor quantum well ${ }^{33}$. In contrast to the conventional Rashba systems, however, we have to take into account of the strong SOC of $\mathrm{SrIrO}_{3}$, the magnetic properties of $\mathrm{SrRuO}_{3}$, and their strong hybridization. Furthermore, since the itinerantelectron systems including $\mathrm{SrRuO}_{3}$ and $\mathrm{SrIrO}_{3}$ have complicated distribution of band-anti-crossings in their band structures $^{22,34,35}$, the quantitative evaluation of the electric fieldinduced modulation in the heterostructures requires elaborate theoretical investigation. Nevertheless, the present observations clearly indicate that the electric field applied to the thin $\mathrm{SrIrO}_{3}$ plays the crucial role in SOC at the interface and even brings about the significant modification of magnetic properties in the neighboring itinerant ferromagnet. The method of inserting a thin nonmagnetic material with strong SOC between a ferromagnet and a gate dielectric may be applicable to tuning many intriguing spin-orbit coupled phenomena such as magnetic anisotropy $^{15}$, domain wall motion ${ }^{36}$ and the DMI iteself ${ }^{37}$.

\section{Methods}

Sample preparation. The epitaxial bilayers composed of $\mathrm{SrIrO}_{3}$ and $\mathrm{SrRuO}_{3}$, and single layer films of $\mathrm{SrRuO}_{3}$ were deposited on $\mathrm{SrTiO}_{3}(001)$ substrates by pulsed laser deposition using a $\mathrm{KrF}$ excimer laser $(\lambda=248 \mathrm{~nm})$. The substrate temperatures during the growth of $\mathrm{SrRuO}_{3}$ and $\mathrm{SrIrO}_{3}$ were $730{ }^{\circ} \mathrm{C}$ and $600{ }^{\circ} \mathrm{C}$, respectively, where oxygen partial pressure was $120 \mathrm{mTorr}$. The laser fluence was $1.2 \mathrm{~J} / \mathrm{cm}^{2}$ for $\mathrm{SrRuO}_{3}$ and $2.6 \mathrm{~J} / \mathrm{cm}^{2}$ for $\mathrm{SrIrO}_{3}$.

Measurement of magnetic and transport properties. The magnetization data were recorded by a SQUID magnetometer with a magnetic field applied perpendicularly to the film plane and along the magnetic easy axis grown on $\mathrm{SrTiO}_{3}(001)$. Magneto-optic Kerr effect was measured with a laser at $690 \mathrm{~nm}$ wavelength in polar geometry by using a photoelastic modulator.

Transport properties were measured in Hall bars cut by a diamond wheel saw $(1 \mathrm{~mm} \times 2.5 \mathrm{~mm})$ and ultrasonically bonded with $\mathrm{Al}$ wires. The applied current was $10 \mu \mathrm{A}$, which corresponds to $3.6 \times 10^{6} \mathrm{~A} / \mathrm{m}^{2}$ for SRO5/SIO2/Sub. Back-gate transistors were fabricated using 0.5 - $\mathrm{mm}$-thick $\mathrm{SrTiO}_{3}$ substrates as a gate dielectric and silver paste as a gate electrode at the opposite side of the deposited films. Antisymmetrizations were performed for both the Hall resistivity and the Kerr rotation angle. Ordinary Hall term was subtracted from the Hall resistivity by linear fitting in a higher magnetic field region.

Data availability. The data that support the findings of this study are available from the corresponding author upon request.

Received: 22 April 2017 Accepted: 14 December 2017

Published online: 15 January 2018

\section{References}

1. Matsukura, F., Tokura, Y. \& Ohno, H. Control of magnetism by electric fields Nat. Nanotechnol. 10, 209-220 (2015).

2. Nagaosa, N., Sinova, J., Onoda, S., MacDonald, A. H. \& Ong, N. P. Anomalous Hall effect. Rev. Mod. Phys. 82, 1539-1592 (2010).

3. Koster, G. et al. Structure, physical properties, and applications of $\mathrm{SrRuO}_{3}$ thin films. Rev. Mod. Phys. 84, 253-298 (2012).

4. Fang, Z. et al. The anomalous Hall effect and magnetic monopoles in momentum space. Science 302, 92-95 (2003).

5. Taguchi, Y., Oohara, Y., Yoshizawa, H., Nagaosa, N. \& Tokura, Y. Spin chirality, berry phase, and anomalous Hall effect in a frustrated ferromagnet. Science 291, 2573 (2001)

6. Nagaosa, N. \& Tokura, Y. Topological properties and dynamics of magnetic skyrmions. Nat. Nanotechnol. 8, 899-911 (2013).

7. Lee, M., Kang, W., Onose, Y., Tokura, Y. \& Ong, N. P. Unusual Hall effect anomaly in MnSi under pressure. Phys. Rev. Lett. 102, 186601 (2009).

8. Neubauer, A. et al. Topological Hall effect in the A phase of MnSi. Phys. Rev. Lett. 102, 186602 (2009).

9. Wang, W. et al. A centrosymmetric hexagonal magnet with superstable biskyrmion magnetic nanodomains in a wide temperature range of 100-340 K. Adv. Mater. 28, 6887-6893 (2016).

10. Mühlbauer, S. et al. Skyrmion lattice in a chiral magnet. Science 323, 915-919 (2009).

11. Yu, X. Z. et al. Real-space observation of a two-dimensional skyrmion crystal. Nature 465, 901-904 (2010).

12. Matsuno, J. et al. Interface-driven topological Hall effect in $\mathrm{SrRuO}_{3}-\mathrm{SrIrO}_{3}$ bilayer. Sci. Adv. 2, e1600304 (2016).

13. Matsuno, J. et al. Engineering a spin-orbital magnetic insulator by tailoring superlattices. Phys. Rev. Lett. 114, 247209 (2015). 
14. Fert, A., Cros, V. \& Sampaio, J. Skyrmions on the track. Nat. Nanotechnol. 8, 152-156 (2013).

15. Weisheit, M. et al. Electric field-induced modification of magnetism in thinfilm ferromagnets. Science 315, 349-351 (2007).

16. Chu, Y.-H. et al. Electric-field control of local ferromagnetism using a magnetoelectric multiferroic. Nat. Mater. 7, 478-482 (2008).

17. Chiba, D. et al. Electrical control of the ferromagnetic phase transition in cobalt at room temperature. Nat. Mater. 10, 853-856 (2011).

18. Valencia, $\mathrm{S}$. et al. Interface-induced room-temperature multiferroicity in $\mathrm{BaTiO}_{3}$. Nat. Mater. 10, 753-758 (2011).

19. Shimizu, S. et al. Gate tuning of anomalous Hall effect in ferromagnetic metal $\mathrm{SrRuO}_{3}$. Appl. Phys. Lett. 105, 163509 (2014).

20. Xia, J., Siemons, W., Koster, G., Beasley, M. R. \& Kapitulnik, A. Critical thickness for itinerant ferromagnetism in ultrathin films of $\mathrm{SrRuO}_{3}$. Phys. Rev. B 79, 140407(R) (2009).

21. Takahashi, K. S., Onoda, M., Kawasaki, M., Nagaosa, N. \& Tokura, Y. Control of the anomalous Hall effect by doping in $\mathrm{Eu}_{1-x} \mathrm{La}_{x} \mathrm{TiO}_{3}$ thin films. Phys. Rev. Lett. 103, 057204 (2009).

22. Nie, Y. F. et al. Interplay of spin-orbit interactions, dimensionality, and octahedral rotations in semimetallic $\mathrm{SrIrO}_{3}$. Phys. Rev. Lett. 114, 016401 (2015).

23. Worledge, D. C. \& Geballe, T. H. Negative spin-polarization of $\mathrm{SrRuO}_{3}$. Phys. Rev. Lett. 85, 5182-5185 (2000).

24. Meynell, S. A. et al. Hall effect and transmission electron microscopy of epitaxial MnSi thin films. Phys. Rev. B 90, 224419 (2014).

25. Machida, Y., Nakatsuji, S., Onoda, S., Tayama, T. \& Sakakibara, T. Timereversal symmetry breaking and spontaneous Hall effect without magnetic dipole order. Nature 463, 210-213 (2010).

26. Ezawa, M. Compact merons and skyrmions in thin chiral magnetic films. Phys. Rev. B 83, 100408(R) (2011).

27. Yu, X. Z. et al. Biskyrmion states and their current-driven motion in a layered manganite. Nat. Commun. 5, 3198 (2014).

28. Koretsune, T., Nagaosa, N. \& Arita, R. Control of Dzyaloshinskii-Moriya interaction in $\mathrm{Mn}_{1-x} \mathrm{Fe}_{x} \mathrm{Ge}$ a first-principles study. Sci. Rep. 5, 13302 (2015).

29. Wakatsuki, R., Ezawa, M. \& Nagaosa, N. Domain wall of a ferromagnet on a three-dimensional topological insulator. Sci. Rep. 5, 13638 (2015).

30. Shibata, K. et al. Towards control of the size and helicity of skyrmions in helimagnetic alloys by spin-orbit coupling. Nat. Nanotechnol. 8, 723-728 (2013).

31. Yasuda, K. et al. Geometric Hall effects in topological insulator heterostructures. Nat. Phys. 12, 555-559 (2016).

32. Toyota, D. et al. Thickness-dependent electronic structure of ultrathin $\mathrm{SrRuO}_{3}$ films studied by in situ photoemission spectroscopy. Appl. Phys. Lett. 87, 162508 (2005)

33. Nitta, J., Akazaki, T., Takayanagi, H. \& Enoki, T. Gate control of spin-orbit interaction in an inverted $\mathrm{In}_{0.53} \mathrm{Ga}_{0.47} \mathrm{As} / \mathrm{In}_{0.52} \mathrm{Al}_{0.48} \mathrm{As}$ heterostructure. Phys. Rev. Lett. 78, 1335-1338 (1997).

34. Chen, Y., Bergman, D. L. \& Burkov, A. A. Weyl fermions and the anomalous Hall effect in metallic ferromagnets. Phys. Rev. B 88, 125110 (2013).

35. Zeb, M. A. \& Kee, H.-Y. Interplay between spin-orbit coupling and Hubbard interaction in $\mathrm{SrIrO}_{3}$ and related Pbnm perovskite oxides. Phys. Rev. B 86, 085149 (2012).
36. Chiba, D. et al. Electric-field control of magnetic domain-wall velocity in ultrathin cobalt with perpendicular magnetization. Nat. Commun. 3, 888 (2012).

37. Nawaoka, K., Miwa, S., Shiota, Y., Mizuochi, N. \& Suzuki, Y. Voltage induction of interfacial Dzyaloshinskii-Moriya interaction in $\mathrm{Au} / \mathrm{Fe} / \mathrm{MgO}$ artificial multilayer. Appl. Phys. Express 8, 063004 (2015).

38. Momma, K. \& Izumi, F. VESTA 3 for three-dimensional visualization of crystal, volumetric and morphology data. J. Appl. Crystallogr. 44, 1272-1276 (2011).

\section{Acknowledgements}

We thank N. Nagaosa, R. Arita, T. Koretsune, and Y. Kaneko for fruitful discussions. This work was partly supported by JSPS KAKENHI Grant Numbers JP24226002, JP17H02791, and JP16J06170 (Y.O.) and by Japan Society for the Promotion of Science through Program for Leading Graduate Schools (MERIT) (Y.O.).

\section{Author contributions}

Y.O., J.M., and M.K. designed the experiments. Y.O. fabricated films, measured transport properties, and analyzed the data. N.O. measured magneto-optic Kerr effect. M.U. and Y. K. contributed to the film fabrication and the transport measurements. Y.O. and J.M. wrote the manuscript. Y.T. and M.K. coordinated the projects. All authors discussed the results.

\section{Additional information}

Supplementary Information accompanies this paper at https://doi.org/10.1038/s41467017-02629-3.

Competing interests: The authors declare no competing financial interests.

Reprints and permission information is available online at http://npg.nature.com/ reprintsandpermissions/

Publisher's note: Springer Nature remains neutral with regard to jurisdictional claims in published maps and institutional affiliations.

(c) (i) Open Access This article is licensed under a Creative Commons Attribution 4.0 International License, which permits use, sharing, adaptation, distribution and reproduction in any medium or format, as long as you give appropriate credit to the original author(s) and the source, provide a link to the Creative Commons license, and indicate if changes were made. The images or other third party material in this article are included in the article's Creative Commons license, unless indicated otherwise in a credit line to the material. If material is not included in the article's Creative Commons license and your intended use is not permitted by statutory regulation or exceeds the permitted use, you will need to obtain permission directly from the copyright holder. To view a copy of this license, visit http://creativecommons.org/ licenses/by/4.0/.

(C) The Author(s) 2018 